From the American

Board of Family Medicine

Ann Fam Med 2020;18:184-185. https://doi.org/10.1370/afm.2526.

\section{WORKING TO ADVANCE THE HEALTH OF RURAL AMERICANS: AN UPDATE FROM THE ABFM}

Over the past 5 decades, existing disparities between the health of rural and urban Americans has grown across a range of outcomes. According to the Centers for Disease Control and Prevention (CDC) ${ }_{1}^{1}$ the 46 million people living in rural America are not only increasingly more likely than urban counterparts to die from each of the top 5 sources of adult mortality-heart disease, cancer, unintentional injury, chronic lower respiratory disease, and stroke-but also experienced a widening gap in child and adolescent death rates between 1999-2017. ${ }^{2}$ James and colleagues describe a "rural mortality penalty," first emerging in the mid-1980s, and rapidly growing higher in the subsequent decades to which 448,000 excess deaths can be attributed by 2007. ${ }^{3}$ Many rural areas have longstanding disparities compared with urban areas in social determinants of health ${ }^{4,5}$ - poverty, intimate partner violence, access to services, economic opportunity, homelessness - and these are often concentrated in rural areas with high percentages of minorities. ${ }^{6}$ New challenges such as the opioid epidemic and increasing multimorbidity have compounded these longstanding disparities in social determinants while access to health care services in rural areas is also in decline. For example, a recent paper found that from 2005 to 2015, the supply of primary care physicians, relative to the population, decreased with rural counties declining more on average than urban counties ( -7.0 per 100,000 population vs -2.6 per 100,000 population). ${ }^{7}$ Additionally, 162 rural hospitals closed between 2005-2019 in an accelerating fashion. ${ }^{8}$ Access to specialty health care services remains a significant challenge for rural populations.

The American Board of Family Medicine (ABFM) and its collaborators have been involved in extensive research to understand the role of Family Physicians (FPs) in order to stem the tide of worsening disparities in this withering ecology of rural health care delivery. Given their ability to care for all populations across all settings, FPs distribute more evenly with the population across the rural/urban continuum than subspecial- ists who require a large referral base or specialized facilities. In relation to other primary care physicians, research using 2010 data found that $80 \%$ of the US population was urban, as well as $90 \%$ of general internists and $78 \%$ of both FPs and general pediatricians. ${ }^{9}$ But general pediatricians were more concentrated in higher population rural area leaving isolated/frontier areas to FPs.

One solution to maintain the presence of FPs in rural areas is training residents in rural settings and ensuring students with rural backgrounds enroll in medical school. Both primary care and rural physician production are concentrated in a small number of academic health centers ("sponsoring institutions"). ${ }^{10}$ Training in an underserved setting is associated with practicing in an underserved setting. ABFM associated research has shown higher rural practice of graduates with increasing rurality of the sponsoring institution $^{10}$ and that training in a federal qualified health center (FQHC), teaching health center, community health center, rural health clinic, or critical access hospital $(\mathrm{CAH})$ were all associated with higher rates of return to these settings. ${ }^{11,12}$ While rural hospitals are closing, the number of $\mathrm{CAH}$ has grown and as of 2009, very few had prior graduate medical education (GME) funding and may be eligible to start training programs..$^{13}$ Student debt is increasing and graduating residents with high debt levels are less likely to intend to practice in underserved settings ${ }^{14}$ or participate in the National Health Service Corps. ${ }^{15}$

Another way to ensure access to care in rural areas is by FPs having a broad scope of practice that not only includes treating chronic and acute conditions and providing procedural care to patients of all age ranges but also providing care in outpatient, inpatient, and emergency settings. Comprehensiveness is a core feature of primary care and past work by Starfield, and ABFM ${ }_{1}^{16}$ has shown when physicians "do more" their patients have better outcomes. Rural FPs practicing more broadly than urban FPs has been demonstrated using both Medicare claims data and self-reported data collected during ABFM examination registration based on the number of services provided and sites where care is delivered. ${ }^{16-20} \mathrm{~A}$ recent analysis found that rural FPs working in patient-centered medical homes had an even higher scope, except for inpatient care and inpatient-related procedures. ${ }^{21}$ By site of care or service delivered, rural FPs provide more maternity care, ${ }^{19,22,23}$ care of children ${ }_{1}^{24}$ women's health ${ }_{1}{ }^{25}$ placement of long-acting reversible contraception and intrauterine devices (LARC/IUDs), ${ }^{26}$ home visits, ${ }^{27}$ mental health care, ${ }^{28}$ endoscopic care, ${ }^{29}$ and emergency department care. ${ }^{30,31}$ Patients located in rural areas are more likely to only see a primary care physician for mental health 
care. ${ }^{32}$ However, scope may be declining in rural areas as evidence shows that over time fewer rural FPs are providing maternity care, ${ }_{1}^{23}$ mental health care, ${ }_{1}^{28}$ endoscopic services, ${ }^{29}$ and women's health. ${ }^{25}$ Recent work found that early-career FPs who intended to provide maternity care at residency graduation had difficulty finding jobs that offered $\mathrm{OB}$, but FPs going rural had a slightly easier time doing so. ${ }^{33}$

The ecology of rural practice for FPs is different than urban practice. Small and solo practices are more common in rural areas. ${ }^{34}$ Rural FPs enjoy higher continuity with their patients. ${ }^{35}$ Rural practices are more likely to have either a nurse practitioner, physician assistant, or certified nurse midwife $i^{36}$ however, the presence of a mental health clinician decreased with increasing rurality. ${ }^{37}$ Rural FPs have been adopting electronic health records at the same rate as urban FPs. ${ }^{38}$

The ABFM remains committed to advancing the health of rural Americans, not only through its ongoing commitment to continuous certification of its diplomates, but also through the advancement of research related to rural America and its primary care workforce. The above findings were produced using ABFM data collected during examination registration of graduating residents and practicing family physicians ${ }^{39}$ and from the National Graduate Survey. ${ }^{40}$ These analyses were done in collaboration with a diverse group of investigators at multiple institutions around the country. The ABFM has been a partner with the University of Kentucky's federally funded Rural and Underserved Health Research Center and has a burgeoning collaboration with The Collaborative for Rural Primary care, Research, Education, and Practice (Rural PREP). Senior ABFM staff are dedicating their time and service to advancing national conversations to impact and improve rural health data, rural GME, and high-performing rural primary care teams, via national advisory service and writing for the Council on Graduate Medical Education Congressional advisory group, the National Academy of Medicine, the Accreditation Council for Graduate Medical Education, and the National Committee for Vital and Health Statistics. As of this writing, ABFM research staff is in discussions with HRSA about how our data can help them evaluate their training programs to better target their dollars to programs with demonstrated outcomes.

The ABFM will continue to support Family Medicine's role in the delivery of health care, and the creation of health in rural America. We have carefully developed and assembled rich data resources through surveys, the PRIME registry, Medicare claims files, and other sources that drive a host of collaborative research efforts characterizing the rural primary care workforce, its training pipeline, and gaps in access to maternity, behavioral, and other dimensions of care as well as potential solutions. Our work to simplify measurement of what really matters in primary care, to ensure payment incentivizes best practices and lowers clinician burnout, is intended to inform pathways to better rural health.

Aware that health care is a minority contributor to overall health, we also must work to better understand and measure social deprivation, as well as the role of FPs and primary care teams in its reduction. Family Medicine can advocate for social policies and regulations that ensure the chances of living a healthy productive life do not depend on accident of the "geography" of birth. Similarly, while FPs are the predominant physician specialty in rural America and practice broadly, we cannot expect to succeed in advancing rural health alone. We must collaborate with other specialty and health professional colleagues. This echoes a call from 30 years ago when ABFM leadership wrote that "family medicine alone should not bear full responsibility for rural health and surgery, psychiatry, OB/gyn, medicine, and pediatrics should contribute for rural training and care." ${ }^{\prime 41}$ And as noted by a team of young leaders convened by the ABFM, ${ }^{42}$ FPs play critical roles in the development of "Communities of Solution," working in multisectoral teams capable of pursuing health and tackling the "problemsheds" that plague rural America. This requires FPs to see their roles as extending beyond the walls of their practices to address broader rural health and social issues, the opioid epidemic and rising rates of suicide being only two of many contemporary examples. The ABFM is committed to these solutions not only through the refinement of certification for rural FPs, but also through research, data collection, and assembly intended to drive conversations relevant to FPs, the patients and communities they serve, and the work and roles that they play in a broader rural landscape. Broad solutions are needed to meet the health care needs of rural areas. Are we up to the challenge?

Lars E. Peterson, $M D, P b D$, Warren P. Newton, $M D, M P H_{i}$ Andrew W. Bazemore, MD, MPH, American Board of Family Medicine, Lexington, KY

References available online only at http:I/www.AnnFamMed.orgl content/18/2/184/suppl/DC1. 